UNIVERSIDADE DE SÃO PAULO

ESCOLA DE EDUCAÇÃO FÍSICA E ESPORTE

\title{
EFEITO DA ESPECIFICIDADE DE ATIVIDADES MOTORAS PRÉVIAS NO CONTROLE MOTOR DE INDIVÍDUOS IDOSOS
}

Juliana Bayeux da Silva

SÃO PAULO

2004

Easy PDF Creator is professional software to create PDF. If you wish to remove this line, buy it now. 
EFEITO DA ESPECIFICIDADE DE ATIVIDADES MOTORAS PRÉVIAS NO CONTROLE MOTOR DE INDIVÍDUOS IDOSOS

\author{
JULIANA BAYEUX DA SILVA
}

\begin{abstract}
Dissertação apresentada à Escola de Educação Física e Esporte da Universidade de São Paulo, como requisito parcial para a obtenção do grau de Mestre em Educação Física.
\end{abstract}

ORIENTADOR: PROF. DR. LUIS AUGUSTO TEIXEIRA

Easy PDF Creator is professional software to create PDF. If you wish to remove this line, buy it now. 